\title{
CUIDADOS DE ENFERMAGEM AO PACIENTE ONCOLÓGICO EM PÓS-OPERATÓRIO DE CIRURGIA DE CABEÇA E PESCOÇO
}

\author{
NURSING CARE TO CANCER PATIENTS IN POSTOPERATIVE PERIOD OF HEAD
}

AND NECK SURGERY

Arieli Cancelier Niero ${ }^{1}$

Maria Salete Salvaro ${ }^{2}$

Neiva Junkes Hoepers ${ }^{3}$

Paula Ioppi Zugno 4

Recebido em: 30 jun. 2017

Aceito em: 12 jun. 2018

RESUMO: As neoplasias malignas em cabeça e pescoço, como o câncer na cavidade bucal, fossa nasal, glândula tireoide e laringe, necessitam de cuidados especiais. Pode-se considerar a enfermagem como uma área importante da oncologia, auxiliando nas atividades preventivas, bem como no processo de tratamento curativo e paliativo do paciente. Desta forma o enfermeiro necessita de conhecimento especializado e habilidades específicas para desenvolver as atividades relacionadas a esta área. O presente trabalho buscou identificar os cuidados de enfermagem necessários aos pacientes oncológicos submetidos às cirurgias de cabeça e pescoço a fim de proporcionar uma adequada recuperação dos pacientes em pós-operatório. Os resultados obtidos referentes às falas dos entrevistados mostram que os profissionais possuem conhecimento acerca dos cuidados prestados aos pacientes, visto que estes estão diretamente relacionados ao bem estar, como a realização do curativo diário, desobstrução de vias aéreas e controle da dor. Por fim, conclui-se que os cuidados de enfermagem corretamente prestados aos pacientes em pós-operatório de cirurgia de cabeça e pescoço são essenciais para a qualidade da assistência e para uma efetiva recuperação pós-cirúrgica. Sendo assim, o enfermeiro deve se fazer presente como um profissional de referência por meio do conhecimento técnico, prático e científico garantindo a assistência adequada aos cuidados específicos que tal complexidade exige e a recuperação do paciente.

Palavras-chave: Câncer. Enfermagem. Cuidados. Cabeça e pescoço.

ABSTRACT: The head and neck malignancies, such as cancer in the oral cavity, nostril, thyroid gland and larynx, require special care. One can consider nursing as an important area of Oncology, assisting in preventive activities, as well as in the process of curative treatment and palliation. In this way the nurse requires specialized knowledge and specific skills to develop activities related to this area. The present study sought to identify the required nursing care to cancer patients subjected to the head and neck surgeries in order to provide an adequate recovery of postoperative

\footnotetext{
${ }^{1}$ Arieli Cancelier Niero - Acadêmica de Enfermagem, Universidade do Extremo Sul Catariennse - UNESC, arieliniero@hotmail.com.

${ }^{2}$ Maria Salete Salvaro - Enfemeira, mestre, docente Universidade do Extremo Sul Catarinense - UNESC, mssalvaro@yahoo.com.br.

${ }^{3}$ Neiva Junkes Hoepers - Enfemeira, mestre, docente Universidade do Extremo Sul Catarinense - UNESC, neivajun@unesc.net.

${ }^{4}$ Paula loppi Zugno - Enfemeira, mestre, docente Universidade do Extremo Sul Catarinense - UNESC, paula33@unesc.net.
} 
patients. The results obtained regarding lines of respondents show that the professionals have knowledge of care to patients, as they are directly related to the well-being, as the realization of the curative daily, clearing Airways and pain control. Finally, it is concluded that the nursing care provided to patients correctly in postoperative period of head and neck surgery are essential to the quality of care and to an effective post-surgical recovery. Therefore, the nurse should do this as a professional reference through the technical, practical and scientific knowledge by ensuring the appropriate assistance to the specific care that such complexity requires and the patient's recovery.

Keywords: Cancer. Nursing. Care. Head and neck.

\section{INTRODUÇÃO}

O câncer é uma das doenças que mais aflige a sociedade atualmente. É caracterizada como um conjunto de patologias heterogêneas, que ocorrem a partir de modificações fundamentais na fisiologia celular, causando assim o desenvolvimento dos tumores malignos (INCA, 2015).

Existem neoplasias benignas e malignas. Nas benignas o crescimento do tumor é mais lento e possui limites que não invadem os tecidos vizinhos, ao contrário dos malignos que podem invadir esses tecidos vizinhos pela corrente sanguínea ou linfática. São multifatoriais as causas das neoplasias malignas, provenientes tanto da predisposição genética quanto do tabaco, álcool e fonte de radiação (BRASIL, 2011).

Os cânceres de cabeça e pescoço, que serão tratados nesta pesquisa, são as neoplasias malignas localizadas nos sítios anatômicos da cavidade oral compreendendo a mucosa bucal, gengivas, palato, língua, soalho da língua, orofaringe, nasofaringe, hipofaringe, cavidade nasal, seios paranasais, laringe e glândulas. Este estudo buscou identificar quais os cuidados de enfermagem podem ser prestados para proporcionar uma adequada recuperação dos pacientes em pós-operatório de cirurgia de cabeça e pescoço.

A partir dos cuidados de enfermagem, tem-se como questão norteadora da pesquisa: quais cuidados de enfermagem podem ser prestados para proporcionar uma adequada recuperação dos pacientes em pós-operatório de cirurgia de cabeça e pescoço?

Com o intuito de desvelar a importância da assistência de enfermagem prestada a esses pacientes, tem-se como objetivo: Identificar os cuidados de enfermagem em pacientes oncológicos submetidos às cirurgias de cabeça e pescoço em um hospital do Sul Catarinense.

\section{MATERIAIS E MÉTODOS}

Trata-se de um estudo de abordagem qualitativa, descritiva, exploratória e de campo, onde o caráter exploratório desta pesquisa caracteriza-se por trabalhar como "universo de significações, motivos, aspirações, atitudes, crenças e valores. Esse conjunto 
de dados considerados qualitativos" condiz a um espaço mais profundo das relações, não podendo reduzir os processos e os fenômenos à operacionalização de variáveis (MINAYO, 2004, p. 28).

O presente estudo foi realizado em unidades de internação e sala de recuperação pós anestésica (SRPA) de um hospital de grande porte do Sul Catarinense. Sendo realizada por quatro enfermeiros e quinze técnicos de enfermagem.

Para a realização da pesquisa os participantes assinaram um termo de consentimento livre e esclarecido, sendo que este assegura o sigilo da identidade dos participantes. O termo segue as exigências formais contidas na resolução $510 / 16$, do Conselho Nacional de Saúde.

\section{RESULTADOS E DISCUSSÕES}

Iniciou-se a coleta de dados com quatro (4) enfermeiros e quinze (15) técnicos de enfermagem de um Hospital do Sul Catarinense. As entrevistas foram realizadas a partir da assinatura do Termo de Consentimento Livre e Esclarecido.

A partir da seleção dos enfermeiros e técnicos de enfermagem, no período da pesquisa realizaram-se as entrevistas com objetivo de conhecer e identificar os cuidados de enfermagem prestados aos pacientes submetidos à cirurgia de cabeça e pescoço.

Para preservar o sigilo decorrente das entrevistas realizadas, de acordo com as diretrizes e normas regulamentadoras da Resolução 510/16 que envolvem a Pesquisa em Ciências Humanas e Sociais com seres humanos, utilizaram-se a letra "E" para enfermeiros e " $T$ " para técnicos de enfermagem, seguido do respectivo número. Inicialmente serão abordados os resultados das entrevistas dos enfermeiros e após os resultados das entrevistas com os técnicos.

\section{PERFIL DOS ENFERMEIROS ENTREVISTADOS}

A idade dos enfermeiros pesquisados variou de trinta e um (31) anos a quarenta e cinco (45) anos. Quanto ao gênero, foi realizada por quatro (4) femininos. O tempo de trabalho como enfermeiro variou de dois (2) anos a doze (12) anos. O tempo de atuação nos respectivos setores entrevistados variou de onze (11) meses a nove (9) anos. Quanto à especialização, os achados encontrados foram: uma (1) com especialização em saúde do trabalhador e pediatria e as três (3) demais não possuem curso de especialização.

Observamos que diante das entrevistadas o tempo de atuação como enfermeira teve variações, bem como o tempo de atuação nos setores. Um dos pontos que nos chama a atenção são os cursos de especialização, sabemos que a enfermagem vem evoluindo de forma significativa na questão do cuidar, consequentemente os cursos de especializações são de suma importância. Diante da complexidade dos cuidados em oncologia nenhuma 
das enfermeiras possui especialização em enfermagem oncológica.

\section{CUIDADOS NO PÓS-OPERATÓRIO E PROTOCOLO DE CUIDADO}

Pode-se observar que a existência de um protocolo específico para os pacientes submetidos à cirurgia de cabeça e pescoço não se faz presente na instituição, perante as respostas dos entrevistados. Porém estes relatam que os médicos lhes orientam e repassam todas as informações referentes aos cuidados a serem realizados com esses pacientes.

Quando questionado sobre a existência do protocolo de cuidado, obteve-se as seguintes respostas:

\footnotetext{
E1 - "Possuímos protocolos clínicos para determinados procedimentos, e também para pacientes paliativos, mas protocolo específico para pós-operatório de cirurgia de cabeça e pescoço desconheço".

E2; E3 e E4 - "Desconheço"
}

Os protocolos são rotinas de ações e cuidados nos serviços de saúde, para orientar fluxos, procedimentos clínicos e condutas a serem realizadas perante uma situação. Esses protocolos são extremamente necessários e úteis para os serviços e processos de trabalho (WERNECK; FARIA; CAMPOS, 2009).

A existência de um protocolo de assistência tem o intuito de sistematizar 0 tratamento e acompanhamento, instrumentalizar a supervisão das ações e subsidiar a educação em serviços de saúde. A utilização deste tipo de ferramenta com base em estudos científicos, é entendida como uma forma de homogeneizar a prática e torná-la mais segura. O seu uso como forma de sistematizar a assistência, possibilita a equipe multidisciplinar avaliar os fatores relacionados aos aspectos clínicos (DANTAS et al., 2013).

Os cuidados de enfermagem aos pacientes com câncer devem ser individualizados, uma vez que o paciente está fragilizado e com uma expectativa de vida reduzida diante do diagnóstico de uma doença neoplásica. Dessa forma, o enfermeiro apresenta-se presente nas transformações fisiológicas e psíquicas, devendo promover uma maior aproximação com o paciente por meio da comunicação, além de identificar as necessidades e proporcionar uma melhor qualidade de vida (PETERSON; CARVALHO, 2011).

A equipe de enfermagem é imprescindível no processo de cuidar dos pacientes oncológicos, envolvendo uma interação de conhecimento e valores a fim de buscar uma relação dinâmica e proporcionar o máximo conforto. Os enfermeiros precisam estar preparados para cuidar dos pacientes, acolhendo-os através da escuta de sentimentos e vivências, amenizando o sofrimento de conviver com a doença e com os efeitos do tratamento (SALIMENA et al., 2013).

As intervenções cirúrgicas se caracterizam por serem procedimentos invasivos e traumáticos para os pacientes. Nesse sentido a enfermagem tem um papel primordial na 
assistência dos pacientes desde a chegada à instituição, a fim de proporcionar melhores condições para o procedimento cirúrgico e garantir menores possibilidades de complicações. Os pacientes, ao serem internados para a realização de uma cirurgia, muitas vezes trazem consigo receios e dúvidas perante o procedimento. Nessa relação, o papel do enfermeiro e da equipe que irá prestar assistência a ele, devem interligar os aspectos humanos no atendimento ao paciente. Por isso é preciso sistematizar os cuidados utilizando conhecimentos teórico-práticos para qualificar a assistência e o acolhimento como estratégias de minimizar o sofrimento (BASTOS et al., 2013).

A cerca dos cuidados de enfermagem no pós-operatório obteve-se as seguintes falas das enfermeiras:

\begin{abstract}
E1 - "Atentar principalmente para as vias aéreas, aspiração traqueal, deixar o paciente sempre com a cabeceira elevada, atentar para as hemorragias, drenos de portovac, higiene em geral, sinais vitais, pois esse paciente pode ter uma hipotensão, medicação para dor, atentar-se também para episódios de náuseas, vômitos, tonturas".

E2 - "Sempre deixar a cabeceira elevada, observar os sangramentos, edema e orientar repouso no leito".

E3 - "Cuidados com sangramento, manter paciente monitorizado nas primeiras 24 horas (oxímetro), observar drenos funcionantes, curativo diário, aspecto da ferida operatória, cuidados com traqueostomia, manter ventilação e aspiração se necessário".

E4 - "Manter sempre a cabeceira elevada, monitorar sinais vitais, realização dos curativos sempre que necessário, avaliar sangramento, observar também se o mesmo está com náuseas e com dor".
\end{abstract}

Sabemos que a enfermagem deve atender as demandas biológicas, sociais, espirituais e psicológicas do paciente, proporcionando assim uma visão holística desde o acolhimento na unidade de internação como também na elaboração do plano de alta, observando sempre as necessidades e prioridades dos pacientes, como também a segurança do paciente (BASTOS et al.,2013).

Diante dos dados pesquisados, observamos que os cuidados no pós-operatório podem ser classificados em mediato ou imediato. Os cuidados mediatos são aqueles realizados desde a entrada do paciente no setor. Já os cuidados imediatos abrangem todas as ações realizadas nas primeiras 24 horas após o ato operatório. 0 enfermeiro que presta assistência aos pacientes submetidos a algum tipo de procedimento cirúrgico devem possuir conhecimentos e habilidades qualificadas para atender esses pacientes. Para tal, o profissional deve planejar os cuidados a serem prestados a fim de recuperar o equilíbrio fisiológico do paciente com o mínimo de complicações e com o propósito de qualificar a assistência prestada (SERRA et al., 2015).

\title{
PARTE SUPERIOR DO FORMULÁRIO
}

\section{CUIDADOS E ORIENTAÇÕES ESPECÍFICAS DO ENFERMEIRO}

Diante desta categoria as falas das enfermeiras evidenciaram o conhecimento 
sobre os cuidados e as orientações a serem repassadas aos familiares e pacientes submetidos à cirurgia de cabeça e pescoço.

Ao enfermeiro, cabe planejar, coordenar e prestar assistência de enfermagem aos pacientes, sendo que os cuidados envolvem alta complexidade e a realização de consulta de enfermagem. Dessa forma, o enfermeiro apresenta-se como elemento central na equipe de saúde. A enfermagem torna-se de grande importância, promovendo o elo entre a equipe de saúde e o vínculo paciente cuidador (ARAUJO, 2008).

\begin{abstract}
E1 - "O enfermeiro fica responsável por realizar o plano de cuidado desse paciente, agendamento de visita da equipe multiprofissional. Ficar atento principalmente às vias aéreas, pois muitas vezes ele necessita de aspiração traqueal [...]. Ter grande cuidado com os ganhos e perdas desse paciente pelo dreno de suctor (portovac), hemorragias que muitas vezes ocorrem por lesões de determinados procedimentos realizados".

E2 - "Realizar visita ao paciente, admissão e acompanhamento diário. Verificar necessidade da troca de curativos e cuidados com os drenos. Cuidados com os pacientes traqueostomizados para que sempre que houver necessidade realizar a aspiração".

E3 - "Orientações aos técnicos de enfermagem em relação aos cuidados, realização de sondagem enteral sempre que solicitado pelo médico, realizar desobstrução da SNE, fazer toalete quando houver obstrução da traqueostomia (rolha)".

E4 - "Orientação ao familiar quanto à deglutição, comunicação, ansiedade e orientar a equipe de enfermagem, quanto aos cuidados específicos".
\end{abstract}

A partir das falas das entrevistadas podemos observar que os cuidados específicos do enfermeiro e suas orientações consistem basicamente na orientação aos técnicos de enfermagem sobre os cuidados a serem realizados principalmente na aspiração traqueal, realização de visitas multiprofissionais como também visitas diárias aos pacientes, orientações quanto à deglutição e cuidados com a sonda nasoentérica.

Desta forma a assistência de enfermagem prestada aos pacientes submetidos às cirurgias possuem grandes variações. Primeiramente, se inicia com a avaliação do nível de consciência, ou seja, pela resposta dos estímulos verbais, e a partir disso se iniciam os demais cuidados, sendo eles: controle dos sinais vitais, padrão respiratório, presença de sangramentos e/ou hemorragias. Sendo assim, o enfermeiro necessita atentar-se para qualquer tipo de intercorrência ou seus sinais, devendo dessa forma agir de maneira rápida e objetiva para prevenir segundos danos ao paciente (SERRA et al., 2015). Ao enfermeiro cabe também ações de orientação aos pacientes, garantindo assim o início de uma assistência humanizada, criando laços de confiança com eles (ROCHA; IVO, 2015).

Cabe ao enfermeiro avaliar e instituir um plano de cuidado individualizado para cada paciente, controlando assim os sintomas apresentados durante o período de internação e orientações para o plano de alta destes, relacionando o nível de dor, o aumento de secreção, sangramento e prurido. O enfermeiro e sua equipe devem avaliar o paciente como um todo (LUCA, 2012).

O enfermeiro que acompanha o contexto da oncologia, demonstra conhecimento dos elementos humanos essenciais para prestar os cuidados mais próximos possíveis com pacientes, envolvendo assim o fortalecimento do vínculo familiar com os profissionais (SANTOS et al., 2013). 


\section{DIFICULDADES ENCONTRADAS}

Observou-se que as dificuldades encontradas perante a assistência para as enfermeiras foram variadas, porém cada uma delas possui seu perfil assistencial e focam em diferentes cuidados.

O enfermeiro, profissional presente em todo o processo de adoecimento, pode contribuir de forma a auxiliar o enfrentamento da doença, no tratamento e também nas situações de risco com os pacientes e familiares, por meio do planejamento de cuidados condizentes com as necessidades e possibilidades de cada paciente. Quando se trata de serviços oncológicos, a prática de enfermagem expõe os profissionais ao contato direto com situações de dor, finitude e morte, além da desesperança de pacientes e familiares, bem como a expectativa de cura da doença (SILVA et al., 2016).

Quando questionadas sobre as dificuldades encontradas, obteve-se as seguintes respostas:

E2 - "Hoje a maior dificuldade é a comunicação entre médicos e a enfermagem, muitas vezes precisamos ligar para os médicos para questiona-los sobre o que deviria estar prescrito, como por exemplo, os cuidados".

E3 - "Manter o paciente e familiares calmos, porem todos os pacientes submetidos a cirurgia da cabeça e pescoço são pacientes ansiosos e agitados".

E4 - "Agitação do paciente devido à ansiedade, e dessa forma provoca sangramentos".

Os enfermeiros têm a responsabilidade de garantir a qualidade dos cuidados prestados, devendo motivar e alertar toda a equipe para uma prática de enfermagem com qualidade. É necessário também que a equipes esteja atenta às necessidades dos pacientes e suas dificuldades para lidarem com os problemas, podendo auxiliar nas situações de estresse que se tornam difíceis de resolver. (SILVA et al., 2016).

E1 - [...] "É saber se o paciente está realizando os devidos cuidados em casa, ter cuidado com a SNE para evitar uma broncoaspiração. Além disso somos muito bem assistidos pela equipe medica frente aos cuidados que deve ter com esses pacientes".

Ao se tratar de oncologia devemos observar que a presença de equipe multiprofissional é de suma importância, composta por profissionais da aérea de fisioterapia, fonoaudiologia, psicologia e nutrição.

O serviço hospitalar é um dos serviços de saúde mais complexos devido aos inúmeros processos administrativos e assistenciais, bem como a implantação das equipes multiprofissionais. $O$ enfermeiro então vivencia diariamente desafios na realização de atividades assistenciais e gerenciais, dessa forma faz parte desse contexto o processo de trabalho para manter e restaurar os níveis de saúde e bem estar dos pacientes, aplicando a sistematização da assistência de enfermagem (SAE) para o planejamento, execução, controle e avaliação dos cuidados diretos prestados a esses pacientes (SOARES et al., 2015).

E1 - "Posso dizer que hoje minha principal dificuldade é saber se o mesmo entrou em contato com a equipe de saúde da unidade básica, e/ou se continuou com o tratamento". 
Diante da fala de E1 podemos observar a importância do sistema de referência e contra referência para a continuidade do tratamento. Desta forma a utilização do sistema de referência e contra referência é uma forma de possibilitar o acesso a todos os serviços vinculados ao SUS, referenciando o atendimento ao serviço especializado ou serviço de referência. Quando finalizado o atendimento, encaminha-se à sua unidade de saúde de origem para a continuidade do tratamento. Esse serviço também serve como lógica de hierarquização dos serviços, com a finalidade de sistematizar os níveis de complexidade de atendimento (BRASIL, 2011).

O vínculo profissional entre médicos e enfermeiros possibilita a realização de um trabalho integrado e cooperativo diante das intervenções realizadas aos pacientes. Desse modo, à valorização interpessoal se faz necessária para compreender a realidade do serviço a fim de promover o crescimento da equipe, possibilitando a diminuição de conflitos (COSTA et al., 2014).

Diante da literatura apresentada pode-se observar que o perfil dos pacientes com neoplasias de cabeça e pescoço em sua maioria são tabagistas, etilistas, do sexo masculino e com idade entre 51 e 60 anos. Perante os diagnósticos esses pacientes ficam transtornados, muitas vezes agitados e agressivos não aceitando a nova aparência física, bem como o novo convívio social. O fumo e o álcool representam maior incidência de câncer para este sítio de lesões uma vez que apresentam em sua formulação componentes mutagênicos e carcinogênicos (PINTO et al., 2012).

\section{ENTREVISTAS COM OS TÉCNICOS DE ENFERMAGEM}

A idade dos técnicos de enfermagem pesquisados variou de vinte e três (23) anos a cinquenta e nove (59) anos. Quanto ao gênero, foi realizada por um (1) masculino e quatorze (14) femininos. O tempo de trabalho como técnico de enfermagem esteve entre dois (2) anos a quatorze (14) anos. O tempo de atuação nos respectivos setores entrevistados variou entre dois (2) dias a seis (6) anos.

Observamos que diante do perfil dos entrevistados percebesse que houve variações, referente ao tempo de atuação nos setores onde muitos estão atuando há pouco tempo. Desse modo o constante aprimoramento, treinamento e capacitação são de suma importância para uma adequada assistência

\section{CUIDADOS NO PÓS-OPERATÓRIO}

A existência de um protocolo de cuidado específico para os pacientes submetidos a cirurgia de cabeça e pescoço não se faz presente na instituição como já observado nas falas. Porém, observa-se que os profissionais prestam assistência adequada aos pacientes, atentos a qualquer intercorrência. 
O quadro a seguir ilustra os cuidados de enfermagem realizados aos pacientes submetidos às cirurgias de cabeça e pescoço.

\begin{tabular}{|c|c|}
\hline Cuidados & Entrevistados \\
\hline Realização de curativo diário & T3; T4; T5; T6; T7; T8; T9; T10; T11; T12; T15 \\
\hline Aspiração traqueal sempre que necessário & T2; T4; T10; T12; T13; T14; T15 \\
\hline Observar sangramento & T4; T5; T8; T10; T11; T11 \\
\hline Observar padrão respiratório/oferta de $\mathrm{O}_{2}$ & T1; T2; T5; T10; T13; T14 \\
\hline Posicionamento do paciente/elevação de cabeceira & T2; T6; T11; T13; T14; T15 \\
\hline Verificação de sinais vitais & T5; T10; T11; T12; T13 \\
\hline Cuidados com drenos & T3; T4; T6; T7; T8 \\
\hline Cuidados com a alimentação/SNE & T3; T4; T6; T8 \\
\hline Amenizar a dor & T3; T6; T7; T9 \\
\hline Monitorização do paciente & T1; T2; T14 \\
\hline Observar edema & $T 5 ; T 10$ \\
\hline Higiene Oral & T3 \\
\hline Atentar para náusea e vômito & T9 \\
\hline
\end{tabular}

Fonte: Dados da pesquisa (2017).

A pesar da não existência de um protocolo de assistência aos pacientes no pósoperatório de cirurgia de cabeça e pescoço, podemos observar no quadro acima que a equipe presta os cuidados de maneira necessária, atentando principalmente para a realização dos curativos, aspiração traqueal, atentar para as emergências oncológicas, oferta de oxigênio sempre que necessário e manter o paciente sempre em posição semifowler.

A equipe de enfermagem tem papel fundamental no cuidado aos pacientes hospitalizados, sendo cuidados no âmbito físico ou psicológico. A enfermagem atua diretamente no cuidado aos pacientes submetidos às cirurgias, que proporcionam maior conforto e a redução de efeitos colaterais causados pelas intervenções (SANTOS et al., 2012).

Quando refere-se aos cuidados no pós-operatório deve-se lembrar das complicações que podem surgir, sendo a dor uma delas, ocasionando dificuldade na mobilização e restringindo assim os esforços nas atividades. Outro exemplo são as hemorragias e sangramentos que apresentam ruptura de ligadura ou hemostasia deficiente. A correta prestação de cuidados sistemáticos aos pacientes permite que essas ocorrências sejam diagnosticadas precocemente a fim intervir de forma rápida e ter sucesso no tratamento e nas correções das complicações (STRACIERI, 2008).

O profissional de enfermagem permanece a maior parte do tempo ao lado do paciente, dessa forma o princípio do cuidado fora das possibilidades terapêuticas deve-se pressupor os cuidados nas dimensões físicas, psíquicas e espirituais a fim da preservação da qualidade de vida do paciente, com a valorização do cuidado emocional traduzido em empatia, promovendo o conforto e bem-estar tanto para o paciente quanto para os familiares (PINTO et al., 2011).

Os cuidados de enfermagem bem como as prescrições médicas, devem ser realizados em conjunto pelos médicos cirurgiões e pelo enfermeiro responsável, já que o 
emprego adequado da SAE é fundamental para o desenvolvimento das rotinas diárias. Além disso, são necessários também para a prestação de cuidados como: manter o paciente sempre em posição semifowler; realizar os curativos diariamente, anotando sempre o aspecto da ferida operatório bem como drenos funcionantes; observar e avaliar sinais vitais e sinais de insuficiência respiratória auxiliando sempre que necessário com $\mathrm{O}_{2}$ (SANTOS, 2010).

\section{CONSIDERAÇÕES FINAIS}

A oncologia tem nos mostrado diariamente o aumento da qualidade de vida e na sobrevida dos pacientes com câncer. Uma das modalidades do tratamento são os procedimentos cirúrgicos, que apesar de muitas vezes serem invasivos apresentam significativos benefícios para esses pacientes, visto que ocorrem em uma área específica.

Os achados da pesquisa referente às falas dos enfermeiros, mostraram que o conhecimento sobre os cuidados de enfermagem perante as cirurgias de cabeça e pescoço consistem em cuidados com os drenos, desobstrução de vias aéreas como a traqueostomia, sonda para alimentação bem como a realização do curativo diário. Já nos relatos dos técnicos de enfermagem, consistem na realização do curativo diário e drenos, aspiração de vias aéreas, emergências oncológicas, controle de sinais vitais bem como amenizar as dores.

Observou-se, através da pesquisa, que os cuidados de enfermagem prestados estão relacionados ao bem estar dos pacientes, através da realização do curativo diário bem como o uso de medicamentos para amenizar as dores. Nesse contexto, a relação da equipe multiprofissional é de suma importância no tratamento e acompanhamento dos pacientes, visto que esses sofrem fortes impactos com o convívio social por conta do sítio anatômico atingido pelas neoplasias.

Diante da complexidade na assistência de enfermagem ao se tratar de pacientes oncológicos bem como os cuidados a serem prestados no pós-operatório de cirurgia de cabeça e pescoço, sugere-se capacitação permanente nas instituições a todos os profissionais que prestam assistência a esses pacientes a fim de possibilita-los uma assistência de qualidade e eficaz, como também a especialização em enfermagem oncológica aos enfermeiros e a elaboração e implantação de um protocolo de cuidados aos pacientes submetidos às cirurgias de cabeça e pescoço.

Deste modo conclui-se que os cuidados de enfermagem prestados aos pacientes em pós-operatório de cirurgia de cabeça e pescoço são essenciais para a qualidade da assistência e para uma efetiva recuperação pós-cirúrgica. Sendo assim, o enfermeiro deve se fazer presente como um profissional de referência por meio do conhecimento técnico, prático e científico garantindo a assistência adequada aos cuidados específicos que tal complexidade exige contribuindo para uma terapêutica holística e com maior qualidade. 


\section{REFERÊNCIAS}

ARAUJO, Cláudia Regina Gomes de; ROSAS, Ann Mary Machado Tinoco Feitosa. O Papel da Equipe de Enfermagem no Setor da Radioterapia: uma contribuição para a equipe multidisciplinar. Revista Brasileira de Cancerologia, Rio de Janeiro, v. 54, n. 3, p.231-237, 2008. Disponível em:

<http://www.inca.gov.br/rbc/n_54/v03/pdf/artigo_4_pag_231a237.pdf>. Acesso em: 15 maio 2017.

BASTOS, Alana Queiroz et al. REFLEXÕES SOBRE CUIDADOS DE ENFERMAGEM NO PRÉ E PÓSOPERATÓRIO: UMA REVISÃO INTEGRATIVA DA LITERATURA. Ciência, Cuidado e Saúde, Maringa, v. 12, n. 2, p.382-390, abr. 2013. Disponível em: <file:///C:/Users/Usuario/Downloads/15724-95850-1-PB.pdf>. Acesso em: 24 maio 2017.

BELHIANE, Heber Paulino Pena; MATOS, Leandro Rodrigo Pereira de; CAMARGOS, Ferreira. O PACIENTE FRENTE AO DIAGNÓSTICO DE CÂNCER E A ATUAÇÃO DOS PROFISSIONAIS DE ENFERMAGEM: UMA REVISÃO INTEGRATIVA DE LITERATURA. Revista de Enfermagem do Centro Oeste Mineiro, Minas Gerais, v. 3, n. 4, p.1374-1381, set. 2014. Disponível em: <file:///C:/Users/Usuario/Downloads/592-39711-PB.pdf>. Acesso em: 29 maio 2017.

BOAVENTURA, Ana Paula; VEDOVATO, Cleuza Aparecida; SANTOS, Francisleine Franck dos. PERFIL DOS PACIENTES ONCOLÓGICOS ATENDIDOS EM UMA UNIDADE DE EMERGÊNCIA. Revista Ciencia y Enfermeria, Chile, v. 21, n. 2, p.51-62, 2015. Disponível em: <http://www.scielo.cl/pdf/cienf/v21n2/art_06.pdf>. Acesso em: 31 maio 2017.

BRASIL. Ministério da Saúde. Instituto Nacional de Câncer. Estimativa 2016: Incidência de câncer no Brasil. Rio de Janeiro: 2015. 126 p.

BRASIL. Ministério da Saúde. Instituto Nacional de Câncer. ABC do Câncer: Abordagens básicas para o controle do câncer. Rio de Janeiro: CEDC, 2011. 127p.

BRASIL. Conselho Nacional de Saúde. Resolução nํ510, de 07 de abril de 2016.

Disponível em: <http://conselho.saude.gov.br/resolucoes/2016/Reso510.pdf>. Acesso em: 07 nov. 2016.

CHRISTO, Zuriel Mello de; TRAESEL, Elisete Soares. ASPECTOS PSICOLÓGICOS DO PACIENTE ONCOLÓGICO E A ATUAÇÃO DA PSICO-ONCOLOGIA NO HOSPITAL. Disc. Scientia. Série: Ciências Humanas, Santa Maria, v. 10, n. 1, p.75-87, 2009. Disponível em: <http://sites.unifra.br/Portals/36/Artigos 2009 CH/06.pdf>. Acesso em: 01 jun. 2017.

COSTA, Juliana Pessoa et al. Resolubilidade do cuidado na atenção primária: articulação multiprofissional e rede de serviços. Saúde Debate, Rio de Janeiro, v. 38, n. 103, p.733743, out. 2014. Disponível em: <http://www.scielo.br/pdf/sdeb/v38n103/0103-1104-sdeb38-103-0733.pdf>. Acesso em: 06 maio 2017.

DANTAS, Daniele Vieira et al. PROPOSTA DE PROTOCOLO PARA ASSISTÊNCIA AS PESSOAS COM ÚLCERAS VENOSAS. Revista de Enfermagem da Ufsm, Santa Maria, v. 3, n. 0, p.618-626, set. 2013. Disponível em: 
<https://periodicos.ufsm.br/reufsm/article/viewFile/11076/pdf>. Acesso em: 17 maio 2017.

DALRI, Rita de Cássia de Marchi Barcellos et al. Carga horária de trabalho dos enfermeiros e sua relação com as reações fisiológicas do estresse. Revista Latino

Americana de Enfermagem, Ribeirão Preto, v. 22, n. 6, p.959-965, nov. 2014. Disponível em: <http://www.scielo.br/pdf/rlae/v22n6/pt_0104-1169-rlae-22-06-00959.pdf>. Acesso em: 09 maio 2017.

LUCA, Manoela Dias de. Necessidades de autocuidado à qualidade de vida de clientes com câncer de cabeça e pescoço: contribuição da enfermagem. 2012. 124 f. Dissertação (Mestrado) - Curso de Enfermagem, Universidade do Estado do Rio de Janeiro, Rio de Janeiro, 2012. Disponível em:

<http://www.bdtd.uerj.br/tde_busca/arquivo.php?codArquivo=3699>. Acesso em: 23 maio 2017.

MINAYO, Maria. Cecília. Souza. O DESAFIO DO CONHECIMENTO: PESQUISA QUALITATIVA EM SAÚDE. V.8. ed. São Paulo: Hucitec, 2004.

PAIVA, Carlos Eduardo et al. O que o Emergencista precisa saber sobre as Síndromes da veia cava superior, Compressão medular e Hipertensão intracraniana. Revista Brasileira de Cancerologia, Rio de Janeiro, v. 54, n. 3, p.289-296, 2008. Disponível em: <http://www.inca.gov.br/rbc/n_54/v03/pdf/revisao_3_pag_289a296.pdf>. Acesso em: 01 jun. 2017.

PETERSON, Aline Azevedo; CARVALHO, Emília Campos de. Comunicação terapêutica na Enfermagem: dificuldades para o cuidar de idosos com câncer. Revista Brasileira de Enfermagem, Brasília, v. 64, n. 4, p.692-697, jul. 2011. Disponível em:

<http://www.scielo.br/pdf/reben/v64n4/a10v64n4.pdf>. Acesso em: 15 maio 2017.

PINTO, Maria Helena et al. O CUIDADO DE ENFERMAGEM AO PACIENTE ONCOLÓGICO FORA DE POSSIBILIDADE DE CURA: PERCEPÇÃO DE UM GRUPO DE PROFISSIONAIS. Cogitare Enfermagem, Paraná, v. 16, n. 4, p.647-653, out. 2011. Disponível em: <file://C:/Users/Usuario/Downloads/25433-92742-2-PB (1).pdf>. Acesso em: 24 maio 2017.

PINTO, Fabio Roberto et al. Tratamento cirúrgico do carcinoma epidermoide da cavidade oral e orofaringe no Instituto do Câncer do Estado de São Paulo (ICESP): perfil dos pacientes tratados e resultados oncológicos iniciais. Revista Brasileira de Cirurgia de Cabeça Pescoço, São Paulo, v. 41, n. 2, p.53-57, abr. 2012. Disponível em: <http://www.sbccp.org.br/wp-content/uploads/2014/11/REVISTA-SBCCP-41-2-artigo01.pdf>. Acesso em: 28 maio 2017.

PINTO, Jose Antônio et al. Lesões pré-malignas da laringe: revisão de literatura. Revista Brasileira de Cirurgia de Cabeça Pescoço, São Paulo, v. 41, n. 1, p.42-47, jan. 2012. Disponível em: <http://www.sbccp.org.br/wp-content/uploads/2014/11/REVISTA-SBCCP41-1-artigo-10.pdf>. Acesso em: 02 jun. 2017.

ROCHA, Débora Rodrigues da; IVO, Olguimar Pereira. ASSISTÊNCIA DE ENFERMAGEM NO PRÉ-OPERATÓRIO E SUA INFLUÊNCIA NO PÓSOPERATÓRIO. Revista Enfermagem Contemporânea, Bahia, v. 4, n. 2, p.170-178, jun. 2015. Disponível em: <file:///C:/Users/Usuario/Downloads/631-3081-1-PB.pdf>. Acesso em: 23 maio 2017. 
RODRIGUES, Luciane Cristine Ribeiro; JULIANI, Carmen Maria Casquel Monti. Resultado da implantação de um Núcleo Interno de Regulação de Leitos nos indicadores administrativo-assistenciais em um hospital de ensino. Einstein, São Paulo, v. 13, n. 1, p.96-102, 2015. Disponível em: <http://www.scielo.br/pdf/eins/v13n1/pt_1679-4508-eins13-1-096.pdf>. Acesso em: 29 maio 2017.

SALIMENA, Anna Maria de Oliveira et al. ESTRATÉGIAS DE ENFRENTAMENTO USADAS POR ENFERMEIROS AO CUIDAR DE PACIENTES ONCOLÓGICOS. Revista de Enfermagem da Ufsm, Santa Maria, v. 3, n. 1, p.8-16, jan. 2013. Disponível em: <file:///C:/Users/Usuario/Downloads/6638-39255-1-PB.pdf>. Acesso em: 17 maio 2017.

SANTOS, Maiara Rodrigues dos et al. DESVELANDO O CUIDADO HUMANIZADO: PERCEPÇÕES DE ENFERMEIROS EM ONCOLOGIA PEDIÁTRICA. Contexto Enfermagem, Florianópolis, v. 22, n. 3, p.646-653, jul. 2013. Disponível em: <http://www.scielo.br/pdf/tce/v22n3/v22n3a10.pdf>. Acesso em: 27 maio 2017.

SANTOS, Daniele Raiane Florentino dos et al. Cuidados de enfermagem ao paciente em pós-operatório de prostatectomia: revisão integrativa. Revista Eletrônica de

Enfermagem, Goias, v. 14, n. 3, p.690-701, jul. 2012. Disponível em: <https://www.fen.ufg.br/fen_revista/v14/n3/pdf/v14n3a27.pdf>. Acesso em: 15 maio 2017.

SANTOS, Nivea Cristina Moreira. Centro cirúrgico e os Cuidados de Enfermagem. 6. ed. São Paulo: Érica Ltda, 2010. 184 p.

SERRA, Maria Aparecida Alves de Oliveira et al. Assistência de enfermagem no pósoperatório imediato: estudo transversal. Escola de Enfermagem Aurora de Afonso Costa, Rio de Janeiro, v. 14, n. 2, p.161-167, mar. 2015. Disponível em: $<$ file:///C:/Users/Usuario/Downloads/5082-24217-1-PB (1).pdf>. Acesso em: 22 maio 2017

SETTE, Catarina Possenti; GRADVOHL, Silvia Mayumi Obana. Vivências emocionais de pacientes oncológicos submetidos à quimioterapia. Rev. Psicol. UNESP, Assis, v. 13, n. 2, p. 26-31, dez. 2014. Disponível em $<$ http://pepsic.bvsalud.org/scielo.php?script=sci_arttext\&pid=S198490442014000200003\&lng=pt\&nrm=iso >. acessos em 22 maio. 2017.

SILVA, Camila Neves da et al. EXERCÍCIO DA LIDERANÇA DO(A) ENFERMEIRO(A) EM UNIDADES ONCOLÓGICAS. Revista Baiana de Enfermagem, Salvador, v. 30, n. 2, p.110, abr. 2016. Disponível em:

<https://portalseer.ufba.br/index.php/enfermagem/article/viewFile/15173/pdf_47>. Acesso em: 18 maio 2017.

SOARES, Mirelle Inácio et al. Sistematização da assistência de enfermagem: facilidades e desafios do enfermeiro na gerência da assistência. Escola Anna Nery Revista de

Enfermagem, Rio de Janeiro, v. 19, n. 1, p.47-53, jan. 2015. Disponível em: <http://www.scielo.br/pdf/ean/v19n1/1414-8145-ean-19-01-0047.pdf>. Acesso em: 27 maio 2017.

STRACIERI, Luís Donizeti da Silva. CUIDADOS E COMPLICAÇÕES PÓSOPERATÓRIAS. Revista da Faculdade de Medicina de Ribeirão Preto, Ribeirão Preto, v. 41, n. 4, p.465-468, 2008. Disponível em:

<http://revista.fmrp.usp.br/2008/VOL41N4/SIMP_4Cuidados_e_complicacoesposoperatorias.pdf>. Acesso em: 24 maio 2017. 
VICENZI, Adriana et al. DADO INTEGRAL DE ENFERMAGEM AO PACIENTE ONCOLÓGICO E À FAMÍLIA. Revista de Enfermagem da Ufsm, Santa Maria, v. 3, n. 3, p.409-417, set. 2013. Disponível em:

<https://periodicos.ufsm.br/reufsm/article/viewFile/8816/pdf>. Acesso em: 24 maio 2017.

WERNECK, Marcos Azeredo Furquim; FARIA, Horácio Pereira de; CAMPOS, Kátia Ferreira Costa. Protocolos de cuidado à saúde e de organização do serviço. Belo Horizonte: Editora Coopmed, 2009. 83 p. Disponível em:

<https://www.nescon.medicina.ufmg.br/biblioteca/imagem/1750.pdf>. Acesso em: 26 maio 2017. 